ON THE ZEROS OF MONOTONE OPERATORS OF RETARDED TYPE IN A BANACH SPACE

by

v. Lakshmikantham

and

Patrick Sutherland

Technical Report No. 30

September 1975 


\section{ON THE ZEROS OF MONOTONE OPERATORS OF RETARDED TYPE IN A BANACH SPACE}

Introduction.

Recent interest in the Cauchy problem for differential equations in a Banach space [9] has stimulated a similar interest for differential equations of a retarded type in a Banach space. The difficulty in imposing assumptions due to a different range and domain space has been overcome in [4] where existence of solutions is established using a monotonicity type condition in terms of norm and weaker forms of differential inequalities.

The theory of existence of solutions of differential equations has been used in $[1,2,5,6,7]$ to obtain existence of zeros and fixed points for nonlinear operators from $E$ into $E$. In this paper we consider the existence of zeros of operators which map into $E$ but whose domain is the space of continuous functions on some real interval into $E$. Since such operators generate differential equations of retarded type, we call such operators retarded type. We employ generalized inner product [1,6] and adapt the methods analogous to $[1,2,7]$. Our results generalize those of [4]. Furthermore, we obtain for the first time, existence of zeros of operators of retarded type. 
1. Preliminary results.

We proceed to define the generalized inner product, $\langle\cdot, \cdot\rangle_{ \pm}: E \times E \rightarrow R$. Let $E^{*}$ be the dual space of $E$. For $x \in E$, set

$$
J(x)=\left\{f \in E^{*}: f(x)=\|x\|^{2} ; \quad\|f\|=\|x\|\right\}
$$

Now for any $y \in E$, define the generalized inner product of $y$ with $x$ as

$$
\langle y, x\rangle_{-}=\operatorname{lnf}_{f \mathrm{e} J(x)} f(y) \text { and }\langle y, x\rangle_{+}=\sup _{f \in J(x)} f(y)
$$

For convenience we list some properties of the generalized inner product.

See $[1,6,9]$ for details.

$$
\left(P_{1}\right)\langle\beta y+\alpha x, x\rangle_{ \pm}=\left\{\begin{array}{l}
\beta\langle y, x\rangle_{ \pm}+\alpha|| x||^{2} \text { if } \beta>0, \\
\beta\langle y, x\rangle_{\mp}+\left.\alpha|| x\right|^{2} \text { if } \beta<0,
\end{array}\right.
$$

$\left(P_{2}\right)\langle x+y, z\rangle_{-} \leq\langle x, z\rangle_{-}+\|y\| \cdot\|z\|$,

$\left(P_{3}\right)$ Let $x:(a, b] \rightarrow E$ be differentiable,

at $t \in(a, b]$. Set $m(t)=\|x(t)\|$,

then $m(t) D_{-} m(t) \leq\left\langle x^{\prime}(t), x(t)\right\rangle_{-}$.

2. Existence and uniqueness of solutions.

Let $\tau>0$ be given and $E$ a real Banach space. Define $E_{0}=C[[-\tau, 0], E]$ the space of continuous functions with norm given by $\|\psi\|_{0}=\max _{-\tau \leq \varepsilon \leq 0}\|\psi(s)\|$

If $t_{0} \in R^{+}$and $x \in C\left[\left[t_{0}-\tau, \infty\right), E\right]$ then for any $t \in\left[t_{0}, \infty\right)$ let $x_{t} \in E_{0}$ be defined by $x_{t}(s)=x(t+s)$ for $-\tau \leq s \leq 0$. Let 
$f \in C\left[R^{+} \times E_{0}, E\right]$. We consider the differential equation of retarded type

$$
x^{\prime}(t)=f\left(t, x_{t}\right)
$$

with the given initial function $\phi_{0}$ e $E_{0}$ at $t=t_{0}$, that is, $x_{t_{0}}=\phi_{0}$. For additional discussion concerning retarded equations see $[4,8]$.

We list the following hypotheses for convenience.

$\left(\mathrm{H}_{1}\right) \quad f \in C\left[R^{+} \times E_{0}, E\right]$

$\left(\mathrm{H}_{2}\right)$ Suppose $A(t)$ is a function from $R^{+}$into $R^{+}$such that $A(t) \neq 0$ for all $t$ and $A(t)$ is bounded on any finite interval. Suppose further

$$
\begin{gathered}
\left\langle D_{-} A(t)[\phi(0)-\psi(0)]+A(t)[f(t, \phi)-f(t, \psi)], \quad[\phi(0)-\psi(0)]\right\rangle_{-} \\
\leq g(t,\|\phi(0)-\psi(0)\| A(t)) \cdot\|\phi(0)-\psi(0)\|
\end{gathered}
$$

whenever $\phi, \psi \in E_{0}$ with

$$
\|\phi(s)-\psi(s)\| A(t+s) \leq\|\phi(0)-\psi(0)\| A(t)
$$

$\left(\mathrm{H}_{3}\right) \quad g \in C\left[R^{+} \times R^{+}, R^{+}\right], g(t, 0) \equiv 0$ and $u \equiv 0$ is the only solution of

$$
\left.u^{\prime}=g(t, u), u\left(t_{0}\right)=0 \text { on } \underline{E}_{0}, \infty\right) \text {. }
$$

$\left(H_{4}\right) \forall T>t_{0}$ and $\varepsilon>0 \exists \delta(T, \varepsilon)>0 \curvearrowright\|f(t, \phi)-f(s, \phi)\|<\varepsilon$ whenever $|t-s|<\delta$

$$
(t, \phi),(s, \phi) \in\left[t_{0}, T\right] \times s_{b}\left(\phi_{0}\right) \text {. }
$$

We need the following known results from $[3,4]$. 
LEMMA 1. Assume that

(a) $g \in C\left[R^{+} \times R^{+}, R^{+}\right]$and $\left[t_{0}, \infty\right)$ is the largest interval of existence of the maximal solution $r\left(t, t_{0}, u_{0}\right)$ of

$$
u^{\prime}=g(t, u) \quad u\left(t_{0}\right)=u_{0} \geq 0
$$

(b) $m \in C\left[\left[t_{0}-\tau, \infty\right), R^{+}\right], S$ is a countable subset of $\left[t_{0}, \infty\right)$ and $\forall t_{1} \geq t_{0}, \quad t_{1} \notin s$ for which $m_{t_{1}}(s) \leq m\left(t_{1}\right),-\tau \leq s \leq 0$. The differential inequality

$$
D_{-} m\left(t_{1}\right) \leq g\left(t_{1}, m\left(t_{1}\right)\right)
$$

holds.

$$
\begin{aligned}
\text { Then if } m_{t_{0}}(s) \leq u_{0},-\tau \leq s \leq 0 \text { we have } \\
m(t) \leq r\left(t, t_{0}, u_{0}\right) \forall t \in\left[t_{0}, \infty\right) .
\end{aligned}
$$

LEMMA 2. Let assumption (a) of LEMMA 1 hold. Suppose that $\left[t_{0}, t_{1}\right] \subset\left[t_{0}, \infty\right)$. Then $\exists \varepsilon_{0}>0$ such that, for $0<\varepsilon<\varepsilon_{0}$, the maximal solution $r\left(t, t_{0}, u_{0}, \varepsilon\right)$ of $u^{\prime}=g(t, u)+\varepsilon, u\left(t_{0}\right)=u_{0}+\varepsilon$ exists on $\left[t_{0}, t_{1}\right]$ and $\lim _{\varepsilon \rightarrow 0} r\left(t, t_{0}, u_{0}, \varepsilon\right)=r\left(t, t_{0}, u_{0}\right)$ uniformly on $\left[t_{0}, t_{1}\right]$.

LEMMA 3. Let $\left(\mathrm{H}_{1}\right)$ hold and $a, b, M>0$ be chosen such that $\|f(t, \phi)\| \leq M$ on $\left[t_{0}, t_{0}+a\right] \times S_{b}\left(\phi_{0}\right)$ where $\phi_{0} \mathrm{e} E_{0}$ is a given initial function. Then for each $k$, a positive integer, there exists an integer $N(\kappa)$, a partition $\left\{t_{i}^{k}\right\}_{i=1}^{N}$ of $\left[t_{0}, t+a\right]$ and a function $x^{k}$ from $\left[t_{0}-\tau, t_{0}+a\right]$ into $E$ with the following properties:

(i) $x_{t_{0}}^{K}=\phi_{0} \quad \forall K$;

(ii) $\left|t_{i+1}^{k}-t_{i}^{k}\right| \leq \frac{1}{k}$ for each $1 \leq i \leq N_{k}$; 
(iii) ||$x_{t}^{k}-x_{s}^{k} \|_{0} \leq M|t-s| \quad t, s \in\left[t_{0}, t_{0}+a\right]$;

(iv) if $t \in\left(t_{i-1}^{k}, t_{i}^{k}\right)$ then $\left(x^{k}\right)^{\prime}(t)$ exists and $\left(x^{k}\right)^{\prime}(t)=f\left(t_{i-1}^{k}, x_{t_{i-1}^{k}}^{k}\right)$ for all $I \leq i \leq N_{k}$;

(v) if $\left\|\phi-x_{t_{i-1}^{k}}^{k}\right\|_{0} \leq M\left(t_{i}^{k}-t_{i-1}^{k}\right)$ then

$$
\left\|f(t, \phi)-f\left(t_{i-1}^{\kappa}, x_{t i-1}^{\kappa}\right)\right\| \leq \frac{1}{k} .
$$

LEMMA 4. Assume the hypotheses of LEMMA 3 and let $x^{\kappa}(t)$ and $\left\{t_{i}\right\}_{i=1}^{N_{K}}$ be the function and partition assured by LEMMA 3. If for each $t \in\left[t_{0}-\tau, t_{0}+\alpha\right]$ we have $x^{k}(t)$ converges pointwise to a function $x(t)$, then $x(t)$ is a solution to the problem

$$
x^{\prime}=f\left(t, x_{t}\right), \quad x_{t_{0}}=\phi_{0} .
$$

We are now in a position to prove an existence and uniqueness result which generalizes the results in [4].

THEOREM 1. Under $\left(\mathrm{H}_{1}\right),\left(\mathrm{H}_{2}\right),\left(\mathrm{H}_{3}\right)$ and $\left(\mathrm{H}_{4}\right)$ the differential equation

$$
x^{\prime}=f\left(t, x_{t}\right), \quad x_{t_{0}}=\phi_{0}
$$

has a unique solution existing on $\left[t_{0}, \infty\right)$.

PROOF. We shall first establish local existence and uniqueness. To this end let $\left\{t_{i}\right\}_{i=1}^{N_{K}}$ be the partitions from LEMMA 3 and observe that 
$S=\bigcup_{\kappa=1}^{\infty}\left\{t_{i}^{\kappa}\right\}_{i=1}^{N_{k}}$ is countable. Set

$$
m^{n, k}(t)=\left\|x^{n}(t)-x^{k}(t)\right\| \cdot A(t)
$$

and note

$$
m_{t}^{n, k}=\left\|\mid x_{t}^{n}-x_{t}^{k}\right\| A(t+8), \quad \text { a e }[-\tau, 0]
$$

If $t_{1} \in\left(t_{0}, t_{0}+a\right]-s$ such that $m_{t_{1}}^{n_{1} k}(s) \leq m^{n_{1} k}\left(t_{1}\right) \neq 0$ for all

$s \in[-\tau, 0]$, then we have

$$
\left\|x_{t_{1}}^{n}(s)-x_{t_{1}}^{k}(s)\right\| A\left(t_{1}+s\right) \leq\left\|x_{t_{1}}^{n}(0)-x_{t_{1}}^{k}(0)\right\| A\left(t_{1}\right) .
$$

Thus

$$
\begin{aligned}
D_{-} m^{n, k}\left(t_{1}\right)= & D_{-}\left(\left\|x^{n}\left(t_{1}\right)-x^{k}\left(t_{1}\right)\right\|\right\} A\left(t_{1}\right)+D_{-} A\left(t_{1}\right)\left\|x^{n}\left(t_{1}\right)-x^{k}\left(t_{1}\right)\right\| \\
\leq & \frac{A\left(t_{1}\right)\left\langle\left(x^{n}\right)^{\prime}\left(t_{1}\right)-\left(x^{k}\right)\left(t_{1}\right), x^{n}\left(t_{1}\right)-x^{k}\left(t_{1}\right)\right\rangle_{-}}{\left\|x^{n}\left(t_{1}\right)-x^{k}\left(t_{1}\right)\right\|} \\
& \quad+D_{-} A\left(t_{1}\right)\left\|x^{n}\left(t_{1}\right)-x^{k}\left(t_{1}\right)\right\| .
\end{aligned}
$$

For $t_{1} \in\left(t_{i-1}^{k}, t_{i}^{k}\right) \cap\left(t_{j-1}^{n}, t_{j}^{n}\right)$, we have 
7

$$
\begin{aligned}
& \left\langle\left(x^{n}\right)^{\prime}\left(t_{1}\right)-\left(x^{k}\right)^{\prime}\left(t_{1}\right), x^{n}\left(t_{1}\right)-x^{k}\left(t_{1}\right)\right\rangle_{-} \\
& =\left\langle f\left(t_{j-1}^{n}, x_{t-1}^{n}\right)-f\left(t_{i-1}^{k}, x_{t_{i-1}^{k}}^{k}\right), x^{n}\left(t_{1}\right)-x^{k}\left(t_{1}\right)\right\rangle_{-} \\
& =\left\langle f\left(t_{j-1}^{n}, x_{t_{j-1}^{n}}^{n}\right)-f\left(t_{1}, x_{t_{1}}^{n}\right)+f\left(t_{1}, x_{t_{1}}^{k}\right)-f\left(t_{i-1}^{k}, x_{t_{i-1}^{k}}^{k}\right)\right. \\
& \left.+f\left(t_{1}, x_{t_{1}}^{n}\right)-f\left(t_{1}, x_{t_{1}}^{k}\right), x^{n}\left(t_{1}\right)-x^{k}\left(t_{1}\right)\right\rangle_{-} \\
& \leq\left[\left\|f\left(t_{j-1}^{n}, x_{t_{j-1}^{n}}^{n}\right)-f\left(t_{1}, x_{t_{1}}^{n}\right)\right\|+\left\|f\left(t_{1}, x_{t_{1}}^{k}\right)-f\left(t_{i-1}^{k}, x_{t k-1}^{k}\right)\right\|\right] \\
& \left\|x^{n}\left(t_{1}\right)-x^{k}\left(t_{1}\right)\right\|+\left\langle f\left(t_{1}, x_{t_{1}}^{n}\right)-f\left(t_{1}, x_{t_{1}}^{k}\right), x^{n}\left(t_{1}\right)-x^{k}\left(t_{1}\right)\right\rangle- \\
& \leq\left[\frac{\underline{1}}{n}+\frac{\underline{1}}{\underline{k}}\right]\left\|x^{n}\left(t_{1}\right)-x^{k}\left(t_{1}\right)\right\|+\left\langle f\left(t_{1}, x_{t_{1}}^{n}\right)-f\left(t_{1}, x_{t_{1}}^{k}\right), x^{n}\left(t_{1}\right)-x^{k}\left(t_{1}\right)\right\rangle-.
\end{aligned}
$$

In obtaining the foregoing inequalities, we have used the properties of generalized inner product and the properties of approximate solutions as given in LEMMA 3.

The last two inequalities, together with $\left(\mathrm{H}_{2}\right)$, yield.

$$
\begin{aligned}
D_{-} m^{n, k}\left(t_{1}\right) \leq A\left(t_{1}\right)\left[\frac{1}{n}+\frac{1}{k}\right] & +\frac{\left\langle A\left(t_{1}\right)\left[f\left(t_{1}, x_{t_{1}}^{n}\right)-f\left(t_{1}, x_{t_{1}}^{k}\right)\right], x^{n}\left(t_{1}\right)-x^{k}\left(t_{1}\right)\right\rangle-}{\left\|x^{n}\left(t_{1}\right)-x^{k}\left(t_{1}\right)\right\|} \\
& +D_{-} A\left(t_{1}\right) \cdot\left\|x^{n}\left(t_{1}\right)-x^{k}\left(t_{1}\right)\right\|
\end{aligned}
$$


8

$$
\begin{aligned}
& \leq A\left(t_{1}\right)\left[\frac{1}{n}+\frac{1}{k}\right]+ \\
& \left\langle D_{-} A\left(t_{1}\right)\left[x^{n}\left(t_{1}\right)-x^{k}\left(t_{1}\right)\right]+A\left(t_{1}\right)\left[f\left(t_{1}, x_{t_{1}}^{n}\right)-f\left(t_{1}, x_{t_{1}}^{k}\right)\right], x^{n}\left(t_{1}\right)-x^{k}\left(t_{1}\right)\right\rangle_{-} \\
& \left\|x^{n}\left(t_{1}\right)-x^{k}\left(t_{1}\right)\right\| \\
& \leq A\left(t_{1}\right)\left[\frac{1}{n}+\frac{1}{k}\right]+g\left(t_{1}, m^{n_{1} k}\left(t_{1}\right)\right) \\
& \leq L\left[\frac{1}{n}+\frac{1}{k}\right]+g\left[t_{1}, m^{n, k}\left(t_{1}\right)\right) \text {, where } L \text { is the bound on }\left[t_{0}, t_{0}+a\right] \text { of } A(t) \text {. }
\end{aligned}
$$

Using LEMMA 1 , we obtain $m^{n, K}(t) \leq r^{n, k}\left[t, t_{0},\left(\frac{1}{n}+\frac{1}{k}\right) \cdot L\right]$, where $r_{n, k}$ is the maximum solution of

$$
u^{\prime}=g(t, u)+L\left(\frac{1}{n}+\frac{I}{k}\right) u\left(t_{0}\right)=L\left(\frac{1}{n}+\frac{1}{k}\right) .
$$

By LEMMA $2, \lim _{n, k \rightarrow \infty} r^{n, k}\left[t, t_{0}, L\left(\frac{1}{n}+\frac{1}{k}\right)\right]=r\left(t, t_{0}, 0\right) \equiv 0$ uniformly on $\left[t_{0}, t_{0}+a\right]$. Hence $\left\{x^{n}(t)\right\}$ converges pointwise and $\lim _{n \rightarrow \infty} x_{n}(t)=x(t)$ is a solution to $x^{\prime}=f\left(t, x_{t}\right), x_{t_{0}}=\phi_{0}$.

To see uniqueness, suppose there are two solutions, $x\left(t, t_{0}, \phi_{0}\right)$ and $y\left(t, t_{0}, \phi_{0}\right) \cdot \operatorname{set}$

$$
m(t)=\left\|x\left(t, t_{0}, \phi_{0}\right)-y\left(t, t_{0}, \phi_{0}\right)\right\| A(t)
$$

Note

$$
m_{t}(s)=\left\|x_{t}(s)-y_{t}(s)\right\| A(t+s) \quad s \in[\tau, 0] \text { and if } t_{1} \in\left[t_{0}, t_{0}+a\right]
$$

such that $m_{t_{1}}(s) \leq m\left(t_{1}\right) \neq 0, \boldsymbol{s \in}[\sim \tau, 0]$ we have

$$
\begin{aligned}
& \left\|x_{t_{1}}(s)\right\| A\left(t_{1}+s\right) \leq\left\|x\left(t_{1}\right)-y\left(t_{1}\right)\right\| A\left(t_{1}\right) \\
& =\left\|x_{t_{1}}(0)-y_{t_{1}}(0)\right\| A\left(t_{1}\right) .
\end{aligned}
$$


Proceeding as before, we obtain

$$
\begin{aligned}
D_{-} m\left(t_{1}\right) & \leq \frac{A\left(t_{1}\right)\left\langle x^{\prime}\left(t_{1}\right)-y^{\prime}\left(t_{1}\right), x\left(t_{1}\right)-y\left(t_{1}\right)\right\rangle}{\left\|x\left(t_{1}\right)-y\left(t_{1}\right)\right\|}+D_{-} A\left(t_{1}\right)\left\|x\left(t_{1}\right)-y\left(t_{1}\right)\right\| \\
& =\frac{\left\langle D_{-} A\left(t_{1}\right)\left[x\left(t_{1}\right)-y\left(t_{1}\right)\right]+A(t)\left[f\left(t_{1}, x_{t_{1}}\right)-f\left(t_{1}, y_{t_{1}}\right)\right], x\left(t_{1}\right)-y\left(t_{1}\right)\right\rangle}{\left\|x\left(t_{1}\right)-y\left(t_{1}\right)\right\|} \\
& \leq g\left(t_{1}, m\left(t_{1}\right)\right),
\end{aligned}
$$

which implies $m(t) \equiv 0, \quad t \in\left[t_{0}-\tau, t_{0}+a\right]$ by LEMMA 1 and $(H 3)$.

Now by local existence $\exists \mathrm{T}>t_{0}$ such that $x\left(t, t_{0}, \phi_{0}\right)$ exists on $\left[t_{0}-\tau, T\right]$. If $T<\infty$ and $\varepsilon>0$ then by $\left(H_{4}\right)$ there exists $\delta\left(T, \varepsilon / L_{1}\right)$, where $L_{1}$ is the bound on $A(t)$ for $t \in\left[t_{0}, T\right]$, such that $\|f(t, \phi)-f(s, \phi)\|<\varepsilon / L_{1}$, whenever $|t-s|<\delta$ and $(t, \phi),(s, \phi) \in\left[t_{0}, T\right] \times s_{b}\left(\phi_{0}\right)$. Let $0<h<\delta$ such that $t_{0}<\mathrm{T}-h$ and define

$$
m(t)=\left\|x\left(t+h, t_{0}, \phi_{0}\right)-x\left(t, t_{0}, \phi_{0}\right)\right\| A(t), \text { for } a z l \quad t \in\left[t_{0}, T-h\right]
$$

Then if for some $t_{1} \in\left(t_{0}, T-h\right)$ such that $m_{t_{2}}(s) \leq m\left(t_{1}\right) \neq 0, s e[-\tau, 0]$ we have

$$
\left\|x_{t_{1}+h}(s)-x_{t_{1}}(s)\right\| A\left(t_{1}+s\right) \leq\left\|x\left(t_{1}+h\right)-x\left(t_{1}\right)\right\| A\left(t_{1}\right)
$$

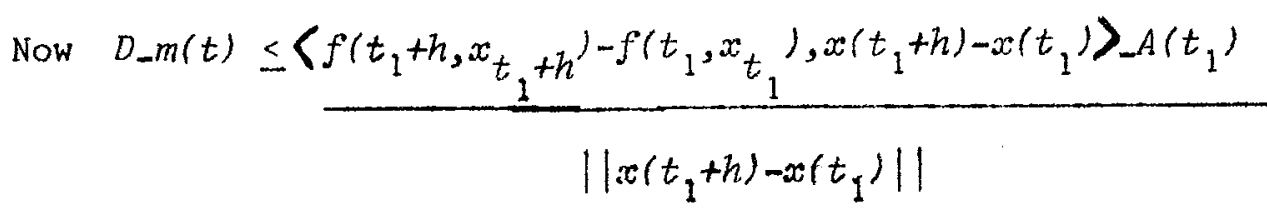

$$
+D_{-} A(t) \cdot\left\|x\left(t_{1}+h\right)-x\left(t_{1}\right)\right\|
$$




$$
\begin{aligned}
& =\frac{A\left(t_{1}\right)\left\langle f\left(t_{1}+h, x_{t_{1}}+h\right)-f\left(t_{1}, x_{t_{1}}+h\right)+f\left(t_{1}, x_{t_{1}+h}\right)-f\left(t_{1}, x_{t_{1}}\right), x\left(t_{1}+h\right)-x\left(t_{1}\right)\right\rangle}{\left\|x\left(t_{1}+h\right)-x\left(t_{1}\right)\right\|} \\
& +D_{-} A\left(t_{1}\right)\left\|x\left(t_{1}+h\right)-x\left(t_{1}\right)\right\| \\
& \leq A\left(t_{1}\right)\left\|f\left(t_{1}+h, x_{t_{1}}+h\right)-f\left(t_{1}, x_{t_{1}}+h\right)\right\|+g\left(t_{1}, m(t)\right) \\
& \leq g\left(t_{1}, m\left(t_{1}\right)\right)+\varepsilon
\end{aligned}
$$

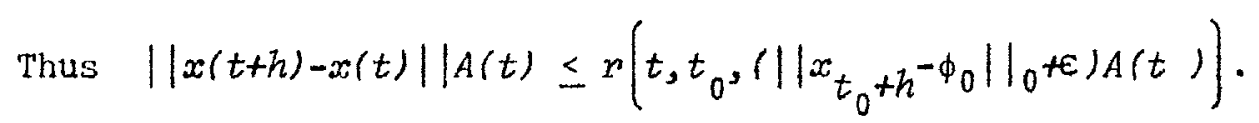

Since $\lim _{u_{0} \rightarrow 0} r\left(t, t_{0}, u_{0}\right) \equiv 0$ and $h \rightarrow 0$ as $t \rightarrow 0$, we have

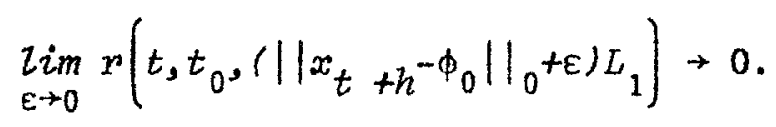

Thus $x\left(t, t_{0}, \phi_{0}\right)$ tends to a limit as $t \rightarrow T$ so we may define $x\left(t, t_{0}, \phi\right)$ for all $t_{\epsilon}\left(t_{0}-\tau, \infty\right)$. Global uniqueness is established in the same fashion as local uniqueness. The proof is therefore complete.

3. Application to zero's of a class of operators of retarded type.

We shall now use the theory of differential equations to investigate the zero's of operators of retarded type. We shall need the following hypothesis on such operators.

$\left(\mathrm{H}_{5}\right) \quad T: E_{0} \rightarrow E$ and $T$ is continuous.

$\left(\mathrm{H}_{6}\right)$ There exists an $A(t)$ with the properties in $\left(H_{7}\right)$ such that $\left\langle A(t)\{T(\phi)-T(\psi)]-D_{-} A(t)(\phi(0)-\psi(0)), \phi(0)-\psi(0)\right\rangle_{+}$ $\geq-g(t,\|\phi(0)-\psi(0)\| A(t))\|\phi(0)-\psi(0)\|$ for $\phi, \psi \in E_{0}$ such that $\|\phi(s)-\psi(s)\| A(t+s) \leq\|\phi(0)-\psi(0)\| A(t)$ for all $s \in[-\tau, 0]$

$\left(\mathrm{H}_{7}\right) A(t)$ is any function from $[-\tau, \infty)$ which has the following properties:

(i) $A(0)=1, \quad A(t)>0 \quad t \in(-\tau, \infty)$ 
(ii) $A$ is bounded on any compact set and $D_{-} A(t)$ exists for all $t \varepsilon(-\tau, \infty)$

(iii) If $r\left(t, 0, u_{0}\right)$ is the maximal solution to $u^{\prime}=g(t, u) \quad u(0)=u_{0}$ then there exists $a t^{*}>0$ and $0<k^{*}<1$ such that $r^{*}\left(t^{*}, 0, u_{0}\right) / A\left(t^{*}\right) \leq k^{*} u_{0}$

$\left(\mathrm{H}_{\mathrm{B}}\right) \quad g \in C\left[R^{+} x R^{+}, R^{+}\right], g(t, 0) \equiv 0$ and $u \equiv 0$ is the only solution of the scalar differential equation $u^{\prime}=g(t, u) \quad u\left(t_{0}\right)=0$. on $\left(t_{0}, \infty\right)$

THEOREM 2. Under assumptions $\left(\mathrm{H}_{5}\right),\left(\mathrm{H}_{6}\right),\left(\mathrm{H}_{7}\right)$, and $\left(\mathrm{H}_{8}\right)$ the operator $T$ has a zero, ie. - there exists a $\phi \in E_{0}$ such that $T(\phi)=0$.

PROOF. Consider the differential equation of retarded type

$$
x^{\prime}+T\left(x_{t}\right)=0 \quad x_{0}=\phi \in E_{0} \text {. }
$$

If we set $f(\phi)=-T(\phi)$, then by $\left(\mathrm{H}_{6}\right)$ we have

$$
\begin{aligned}
& A(t)(f(\phi)-f(\phi))+D \_A(t)(\phi(0)-\psi(0)), \phi(0)-\psi(0)- \\
\leq & g(t,\|\phi(0)-\psi(0)\| A(t)) \cdot\|\phi(0)-\psi(0)\|,
\end{aligned}
$$

whenever $\phi, \psi \varepsilon E_{0}$ such that $\|\phi(s)-\psi(s)\| A(t+s) \leq\|\phi(0)-\psi(0)\| A(t)$ for all $s \varepsilon(-\tau, 0)$. Since $T$ is continuous, $f$ is continuous. Also $f$ aatisfies $\left(\mathrm{H}_{4}\right)$ trivially. Consequently THEOREM 1 assures global existance and uniqueness. Now let $\phi \in E_{0}$ and $x(t, 0, \phi)$ be the solution to $x^{\prime}+T\left(x_{t}\right)=0 x_{0}=\phi$. Define the operator $U(t): E_{0} \rightarrow E_{0}$ as $U(t)(\phi)=x_{t}(0, \phi)$. Because of the autonomous nature of the differential equation under consideration we have that $U(t+s)(\phi)=U(t)[U(s)(\phi))=U(s)[U(t)(\phi))$. Now let $m(t)=\|x(t, \phi)-x(t, \psi)\| A(t)$. Proceeding as before we obtain $m(t) \leq r\left(t, 0,\|\phi-\psi\| \|_{0}\right), t \geq 0$, where $r(t)$ is the maximal solution of $u^{\prime}=g(t, u), u(0)=|| \phi-\psi \|_{0}$. 
As a result, we have $\|x(t, \phi)-x(t, \psi)\| A(t) \leq p\left(t, 0,\|\phi-\psi\| \|_{0}\right), \quad t \geq 0$.

Hence if $t=t^{*}$ in $\left(\mathrm{H}_{7}\right)$ we have

$$
\left\|U\left(t^{*}\right)(\phi)-U\left(t^{*}\right)(\psi)\right\|_{0}=\left\|x_{t^{*}}(0, \phi)-x_{t^{*}}(0, \psi)\right\|_{0} \leq k^{*}\|\phi-\psi\|_{0}
$$

which implies $U\left(t^{*}\right)$ is a contraction. Hence $U\left(t^{*}\right)$ has a fixed point $\phi^{*}$, that is, $\phi^{*}=U\left(t^{*}\right)\left(\phi^{*}\right)$. But

$$
\begin{aligned}
\left\|U(t) \phi^{*}-\phi^{*}\right\|_{0} & =\left\|U(t)\left(U(t)\left(\phi^{*}\right)\right)-U\left(t^{*}\right)\left(\phi^{*}\right)\right\|_{0} \\
& =\left\|U\left(t^{*}\right)\left(U(t)\left(\phi^{*}\right)\right)-U\left(t^{*}\right)\left(\phi^{*}\right)\right\|_{0} \\
& \leq k^{*}\left\|U(t)\left(\phi^{*}\right)-\phi^{*}\right\|_{0}
\end{aligned}
$$

Thus $x_{t}\left(0, \phi^{*}\right)=\phi^{*}$ for all $t$ which implies $\phi^{*}$ is a constant function, $x^{\prime}\left(t, 0, \phi^{*}\right)=0$ and $T\left(\phi^{*}\right)=0$. The proof is therefore complete.

REMARKS. An important special case of $\left(H_{6}\right)$ is when $g \equiv 0$ and $A(t)=e^{\propto t}, \infty>0$. In this case the hypothesis reduces to the form

$$
\begin{aligned}
& \langle T(\phi)-T(\psi)-\alpha(\phi(0)-\psi(0)), \phi(0)-\psi(0)\rangle_{+} \geq 0 \text { for } \phi, \psi \varepsilon E_{0} \text { such that } \\
& \|\phi(s)-\psi(s)\| e^{\alpha s} \leq\|\phi(0)-\psi(0)\| \text { for all } s E(-\tau, 0) \text {. Note that } e^{\alpha t}
\end{aligned}
$$

has the desired propenties of $\left(H_{7}\right)$. For this case, the operator $T$ is said to be strongly monotone. If further $\propto=0$, then $T$ is said to monotone. If $\tau=0$, these definitions reduce to the usual definitions of operators which map $E$ into $E$. 


\section{REFERENCES}

1. K. Deimling, Zeros of Accretive Operators. To appear in Manuscripta Math.

2. V. Lakshmikantham and S. Leela, On the Existance of Zeros of Lyapunov-Monotone Operators. (To appear in Journal of Applied Math and Computation.)

3. V. Lakshmikantham and S. Leela, Differential and Integral Inequalities, Volume II, Academic Press, New York, 1969.

4. V. Lakshmikantham, A. R. Mitchell and R. W. Mitchell, on the Existance of Solutions of Differential Equations of Retarded Type in a Banach Space, (To appear).

5. R. A. Martin, Jr., Lyapunov Functions and Autonomous Differential Equations in a Banach Space, Math Sys. Theory 7 (1973), 66-72.

6. R. H. Mantin, Jr., Differential Equations on Closed Subsets of a Banach Space, Tlans. Amer. Math. Soc., 179 (1973), 399-414.

7. G. Vidomich, How to Get Zeros of Monotone and Accertive Operators Under the theory of Ordinary Differential Equations. (To appear in Aetas Sem. Anal. Func. Sao Paulo.

8. G. Ladas and V. Lakshmikantham. Differential Equations in Abstract Spaces. Academic Press, New York, 1972.

9. V. Lakshmikantham. Stability and Asymptotic Behaviour of Solutions of Differential Equations in a Banach Space. C.I.M.E., Lecture Notes, Cremonese, Rome, 1974. 